\title{
TONIGHT WE RIOT. REPRESENTACIÓN Y CONTROL VIDEOLÚDICA DE LA MULTITUD
}

\section{TONIGHT WE RIOT. CROWD REPRESENTATION AND CONTROL ON VIDEO GAMES}

\author{
Antonio Flores Ledesma \\ Universidad de Granada. Granada / España \\ a.floresledesma@gmail.com \\ https://orcid.org/0000-0002-9616-6683
}

Recibido/Received: 23/06/2020

Modificado/Modified: 14/10/2020

Aceptado/Accepted: 05/11/2020

\section{RESUMEN}

La multitud se ha convertido en un elemento político importante en la actualidad, y el videojuego ha recogido el testigo y se ha encargado de representarla y de hacerla un personaje más en sus obras. En el presente trabajo se van a abordar una serie de obras recientes que implementan a la multitud como personaje jugable para observar el modo en nos representamos como colectivo en ellas. No sólo hay que tener en cuenta el avance técnico que supone la representación dinámica de una pluralidad de individuos que se mueven conjuntamente, sino también -y sobre todo- la forma en que se representa a la multitud como sujeto político, que es, a fin de cuentas, una representación de la realidad.

\section{PALABRAS CLAVE}

Multitud; violencia; cuerpo político; representación; turba.

\section{SUMARIO}

1. Introducción. 2. Marco teórico. 2.1. Idea de Multitud. 2.2. Representación de la multitud en el videojuego. 3. Objetivo y materiales. 4. La multitud en el videojuego. 4.1. Multitud liderada. 4.2. Multitud agente. 5. Contradicciones de la representación de la multitud. 5.1. El espacio y la lucha. 5.2. El cuerpo político. 6. Conclusiones. Bibliografía y Ludografía.

\begin{abstract}
The crowd has become an important political element today, and the video game has collected the witness and has been in charge of representing it and making it one more character in its works. In this paper, will be addressed a few of recent works that implement the crowd as a playable character to observe the way we represent ourselves as a collective in them. It is not only necessary to take into account the technical progress involved in the dynamic representation of a plurality of individuals who move together, but also - and above all - the way in which the multitude is represented as a political subject, which is, ultimately, a representation of reality.
\end{abstract}

\section{KEYWORDS}

Crowd; Violence; Political Body; Representation; Mob. 


\section{CONTENTS}

1. Introduction. 2. Theoretical framework. 2.1. Idea of Crowd. 2.2. Crowd representation on video games.

3. Target and materials. 4. Crowd on video games. 4.1. Led crowd. 4.2. Agent crowd. 5. Contradictions on crowd representation. 5.1. Space and struggle. 5.2. Political body. 6. Conclusions. References and Ludography.

\section{INTRODUCCIÓN}

La "multitud" es algo más que una gran cantidad de gente. A partir del asentamiento de la Revolución Industrial en Europa, en las populosas metrópolis, la multitud toma forma como agrupación heterogénea de personas que ocupan el espacio público de forma activa, con intereses individualizados y proyectos bien diferenciados que co-existen y conviven, y a veces se unen en proyectos comunes. Durante el último tercio del siglo XX, y especialmente desde la crisis de 2007, la multitud ha tomado lugar en el escenario político y social como un sujeto propio.

La multitud se ha convertido en un sujeto para proyectos políticos concretos, y es algo que también ha interesado a la cultura. El videojuego ha permitido, por sus características, no sólo representar a la multitud, si no también formar parte de ella, y esto obliga preguntarse qué es lo que se representa cuando se representa a una multitud controlable en el videojuego. En este caso, cómo expresa la multitud como sujeto político o, sencillamente, qué es lo que dice de ella.

\section{MARCO TEÓRICO}

\subsection{Idea de multitud}

El concepto de "multitud" tiene un largo recorrido, aunque no haya sido hasta hace relativamente poco cuando se ha vuelto popular. Paolo Virno, en La gramática de la multitud (2003), busca su origen en Hobbes y Spinoza, y la contraposición entre ambos a la hora de concebir el "cuerpo político" en la constitución del Estado. El Leviatán de Hobbes, en el establecimiento del "poder", busca el "monopolio de la violencia" (expresión de Max Weber) y la "unificación de la voluntad", y para eso se vale de la idea de "pueblo" como unidad orgánica de una población en un único destino (trascendental, espiritual) que la "cohesiona". La postura de Hobbes determina las relaciones entre un poder que aparece como autoritario y la "ciudadanía" -aquellos que componen el pueblo-, como una referencia del poder vaciada del mismo, pero necesaria para su mantenimiento.

En oposición, la postura de Spinoza tiende a lo que hoy se puede entender generalmente como "democracia": un cuerpo político que es capaz de mantener una heterogeneidad sin que esto afecte a la unidad política como actividad común. Esto es lo que Spinoza llama "multitud", es decir, "una pluralidad que persiste como tal en la escena pública, en la acción colectiva, en lo que respecta a los quehaceres comunes - comunitarios-, sin converger en un Uno, sin desvanecerse en un movimiento centrípeto" (Virno, 2003: 21). Es la forma de existencia social y política de los "muchos en tanto muchos". En la "construcción del poder" tiene sentido el recelo de Hobbes con respecto a la multitud, porque ésta "es refractaria a la obediencia, no establece pactos durables, no consigue jamás el estatuto de persona jurídica porque nunca transfiere los propios derechos naturales al soberano" (Virno, 2003: 23). Es decir, la multitud 
conforma un poder no domesticable, incontrolable por una "autoridad".

Este rechazo ayuda a entender lo que George Rudé (2003) llama multitud "pre-industrial". En la Modernidad, la multitud se articula principalmente como agente político de revuelta cuando las condiciones de vida se ven amenazadas, sea por el hambre, los impuestos, las guerras, etc. Rudé señala que quienes componían la multitud "fueron las 'clases bajas' o menu peuple de las ciudades y la campiña o aquellos que, en París y otras ciudades durante la Revolución Francesa, fueron llamados sans-culottes" (2009: 242, cva. org.). Pequeños artesanos, trabajadores gremiales, etc., es decir, un reflejo de las pautas sociales preindustriales que tenían reclamaciones políticas (sociales, económicas, ...), muy concretas, y más dependientes de las condiciones materiales específicas que de un objetivo político a largo plazo.

La concepción más popular de multitud ha sido la desarrollada por Toni Negri y Michael Hardt. Se basan en Spinoza, del que extraen la idea de multitud como "contrapoder" frente al Leviatán hobbesiano. La multitud sería la articulación política a largo plazo de esa "multitud pre-industrial" -que sólo se unía en momentos de crisis-, es decir, la configuración de una pluralidad política en una corriente más o menos orientada pero nunca unitaria: "La multitud desafía la representación porque es una multiplicidad ilimitada e inconmensurable" (Negri y Hardt, 2002: 162). La multitud como multiplicidad singular, como universal concreto o, en una formulación más netamente marxista, como "unidad no coactiva de lo múltiple" (Wellmer, 1993).

La multitud se diferencia del "pueblo" en que no tiene esa unidad trascendental basada en origen, lengua, la "raza" u otros rasgos diferenciales. También se diferencia de la "masa", la agrupación homogeneizada de la población, un pueblo "sin atributos", que es la forma que éste toma bajo el capitalismo avanzado. El vaciado de "las masas" de sentido las vuelve una fuerza "irracional”, pasiva y, según Negri y Hardt, "fácil de manipular"; mientras, "la multitud es por el contrario un agente social activo, una multiplicidad actuante. No constituye una unidad, como el pueblo, pero a diferencia de las masas está organizada" (2002: 162). La multitud es una forma de resistencia de la población de convertirse en "masa" o "pueblo" por parte de la acción del Estado -el Imperio- (Negri y Hardt, 2004: 129). Por eso, para estos autores, la multitud no existe sin proyecto político (que buscaría a una "verdadera democracia" como forma de expresión de las múltiples singularidades de la multitud).

La multitud aparece en el contexto presente como un sujeto político nuevo, acondicionado a un sistema complejo donde las distinciones como la "clase" se han vuelto más difusas -que es la base del análisis post-marxista Negri y Hardt-. Se dan una serie de condiciones de cooperación e hibridación que recorren transversalmente la sociedad, abriendo nuevas formas de relación social y con el poder -una postura que ha sido cuestionada por su optimismo político (Hauerwas, 2003; Dean y Passavant, 2003; Thoburn, 2003)-. La forma que ha tomado la multitud en el imaginario colectivo se ha establecido como la forma de generar un espacio de comunicación social alternativo. Cuando se "reúne una multitud" se amplía la visión de la "turba" y se excluye a la "masa": desarrolla una intención política de largo alcance.

La multitud tampoco puede devenir masa desde el momento en que es una composición de individualidades. No es un lugar donde se aglutina una "voluntad general", sino la cohesión de voluntades diversas con diferentes grados de integración colectiva, o, como dice Virno, "la multitud contemporánea no está compuesta ni por 'ciudadanos' ni por 'productores'. Ocupa una región intermedia entre lo 'individual' y lo 'colectivo"' (2003: 25). Tal vez esta es la mayor dificultad para representar la multitud: la multitud en un sentido político proyectivo atiende a una nueva "ecología organizativa" que trata de superar esquemas tradicionales (partidos, clases, movimientos) para articular la pluralidad de la sociedad (Williams y Srnicek, 2015: 223 
y ss.), pero lo hace con un lenguaje anclado en esos esquemas.

Esto se ha desarrollado con fuerza a partir de la crisis económica de 2007. Se ha dado una "activación" de la multitud con estas fórmulas de pluralidad y transversalidad, y se ha manifestado en fenómenos como Occupy, el 15M o la "Primavera árabe", y otros movimientos de ámbito más local (de la PAH a Gamonal o Murcia), al mismo tiempo que se ha dado un refuerzo del Estado represivo (Oliver y Urda, 2015). Se habla de organización a través del horizontalismo, democracia directa, y el carácter particular de los diferentes movimientos sociales a partir de 2008, mucho más organizados en Europa, por la presencia de partidos, que, por ejemplo, Occupy en los Estados Unidos (Williams y Srnicek, 2015: 39 y ss.). Es aquí, en estos fenómenos, a partir de los cuales se centra el interés creciente de la representación de la multitud.

\subsection{Representación de la multitud en el videojuego}

La representación de grandes grupos de "personajes no-jugadores" [Non-player character (NPC)] -en oposición al "personaje jugador" [player character (PC)]- ha seguido, por lo general, un desarrollo centrado en el "realismo" de los mundos videolúdicos. La generación de una multitud [crowd] tiene como objetivo dotar al escenario de una sensación de vida, de estar poblado por seres que tienen voluntad y destino dentro del mundo que se comparte, y no son mero atrezzo y escenografía. En este orden, la programación y desarrollo de "mundos" y "poblaciones" que aparentemente se conducen de forma independiente a lo que realiza el PC se ha perfeccionado, de forma que hoy ya se construyen escenarios "creíbles" que permiten un alto grado de inmersión.

Las principales investigaciones sobre la simulación de la multitud [crowd simulation] se han centrado en un primer momento en la apariencia y la ilusión de variedad (McDonnell et al., 2008; Maïm et al., 2009), así como en la simulación realista de la misma en torno a su comportamiento e interacción con el PC (Ondřej et al., 2010). Acerca de esto, en un estudio sobre simulación de multitudes y la interacción de individuos con éstas en un medio de realidad virtual (Bruneau et al. 2015), se concretó que los individuos, por lo general, prefieren evitar las multitudes si se encuentran con ellas, rodearlas, independientemente del carácter de éstas. Este aspecto es especialmente relevante, porque no sólo implica la forma en que el usuario se relaciona con la multitud sino también a través de qué reglas.

El comportamiento de y con la multitud condicionado por las reglas del videojuego reconfigura el comportamiento del usuario con su PC (entendido, de momento, como protagonista individual). Por ejemplo, en el caso de 1979 Revolution: Black Friday (iNK Stories, 2016), el protagonista es un fotoperiodista en medio de la revolución iraní. Es, en principio, un observador pasivo de la multitud, que se presenta variada y viva en un contexto muy concreto. El PC se configura como un testigo que tiene que sumergirse en la multitud (para documentarla), sin llegar a pertenecer nunca a ella. En otros casos, sobre todo en títulos de acción con un componente violento fuerte, las reglas establecen otras dinámicas. En Bloodborne (From Software, 2015) abundan las "turbas" de NPC's enajenados y violentos. Quien juega tiene que sortearlas y urdir estrategias que le permitan enfrentarse a los miembros de las turbas en solitario, para reducir su amenaza.

Más interesantes son aquellos videojuegos en los que la multitud supone una mecánica más de la que el PC individual se puede aprovechar. Se da especialmente en aquellos títulos que tienen un componente de "sigilo" o "infiltración". En sagas como Hitman (IO Interactive) o Assassin's Creed (Ubisoft), los PC's tienen como misiones principales el asesinato de NPC's concreto bajo unas condiciones específicas. Para ello se tienen que valer de las diferentes herramientas que aporta el videojuego, y una de ellas es la multitud. El PC puede ocultarse y 
confundirse con la multitud si es perseguido para pasar desapercibido, o usarla como disfraz para acercarse a su víctima sin ser visto. En ambos casos, la multitud es una herramienta pasiva que se encuentra en el escenario y que sólo accidentalmente se ve implicada en la acción.

El paso de la multitud "como escenario" a la multitud "como personaje" (jugable, agente) tiene que ver con la capacidad de identificación con el usuario y su capacidad de acción. En Hitman o Assassin's Creed se puede formar parte de la multitud, pero en ningún caso ser de la multitud. En las batallas simuladas de la saga Total War (Creative Assembly), las unidades militares están compuestas por decenas de unidades que tienen un comportamiento dinámico en el contexto de una batalla. Pero no se da un control directo, una agencia como PC: el usuario se identifica como "general", no como soldado de una unidad concreta que participa en la acción de esa unidad. Sin embargo, el proceso de identificación se abre en ese espacio. De lo que se trata es de introducir la conciencia de quien juega en la multitud.

Desde la psicología social se ha sugerido que la identificación del usuario con el PC tiene que ver con el disfrute del videojuego (Christoph et al., 2009; Hefner et al., 2007), y que suele tener mayor incidencia cuando se genera una relación con el PC a través de una forma de empatía. En el caso de la multitud se hablaría de "pertenencia". Junto con las necesidades técnicas se encuentran las exigencias de contenido, es decir, no sólo que el control de la multitud se ajuste a los parámetros de comportamiento individual dentro de un colectivo unitario, sino que el control sea desde dentro de la multitud -como ella o como parte de ella- y que se den unas dinámicas efectivas de multitud. La finalidad es permitir que el usuario sienta que tiene el control de una pluralidad como parte de ella y que ésta está sujeta a inercias por las cuales es capaz de actuar por sí misma; que sienta que es todo y parte al mismo tiempo.

\section{OBJETIVO Y MATERIALES}

El objetivo de este trabajo es analizar las diferentes estrategias de representación de la multitud (controlable desde dentro, "agente") en el videojuego como sujeto político en los términos ya señalados, y discernir su capacidad expresiva como figura de una reivindicación política y social concreta. En particular, cómo en los títulos seleccionados la multitud representa o no lo que pretende, o queda más bien como representación de "turba" o "muchedumbre" sin ser capaz de atender a las exigencias conceptuales de la idea de multitud. Lo fundamental de estos títulos es que la multitud sea agente a través de las acciones del usuario, es decir, donde activamente la multitud constituye un PC.

Se han excluido títulos en los cuales sí son centrales las dinámicas de multitudes pero sus acciones no son determinadas por el usuario, sino que responden a unas condiciones preestablecidas donde el usuario sólo planifica, no controla. Es el caso, por ejemplo, de títulos como Ultimate Epic Battle Simulator (Brilliant Game Studio, 2017) o Totally Accurate Battle Simulator (Landfall Games, 2019), en los cuales se puede componer un grupo de entidades con comportamientos dinámicos que se van a enfrentar -en el contexto de una batalla-, pero una vez se le da al play lo elegido sigue una dinámica independiente de las decisiones del usuario.

Tampoco se tienen en cuenta aquellos títulos donde el interés por el "control colectivo" se hace desde la perspectiva y crítica de la "masa". Ejemplo de esto puede ser Kids (Playables, 2019), donde una masa de individuos homogéneos responde a las interacciones del usuario con dinámicas que se pueden juzgar "irracionales" como crítica al comportamiento de las masas; algo similar ocurre con Inside (Palydead, 2016) donde en ocasiones se toma el control de humanos desprovistos de voluntad y centra su crítica en la deshumanización por parte del 
sistema. El interés de este trabajo, por lo tanto, se centra en la conducción agente de la multitud, no simplemente en la composición general y comportamiento en el videojuego.

Para ello se han seleccionado videojuegos donde la multitud, en un primer momento simplemente como agrupación de individuos, constituye el personaje jugable principal. Posteriormente se evaluará su ajuste a las características de la multitud como sujeto político: a) como la unión de una pluralidad heterogénea con diferentes dinámicas dentro del mismo colectivo (lo que le diferencia del "pueblo"); b) la articulación de la multitud como "contrapoder", como una forma de resistencia contra un poder dominante -opresivo- dentro del mundo videolúdico (lo que le diferencia de la "masa"); y c) la configuración en torno a unas reivindicaciones de carácter político, social, económico, etc., de tipo estructural, que van más allá de la mera violencia y destrucción del entorno (lo que le diferencia de la "turba").

Sólo se ha establecido una distinción dentro de las mecánicas de control de la multitud: cuando dentro de la multitud hay una entidad controlada directamente por el usuario que hace las veces de eje o guía de la multitud, y cuando la multitud actúa como cuerpo unitario sin que ninguna entidad destaque por control o agencia por parte del usuario. Lo que se pretende observar, más allá de esta distinción que implica el modo en que el usuario se relaciona con la multitud, es ver cuáles son las dinámicas de la multitud en torno al espacio que ocupa, a su relación con las reivindicaciones que actúan como contexto a la acción, y a la forma de las relaciones consigo misma como pluralidad cohesionada.

\section{LA MULTITUD EN LOS VIDEOJUEGOS}

\subsection{Multitud liderada}

Es discutible que el "liderazgo" pueda formar parte de la multitud, y ha sido debatida su aparición en los movimientos sociales que buscan el horizontalismo como una forma de dirigir -y, en cierto sentido, "pervertir"- la democracia directa en casos como el 15M (Allen-Perkins Avendaño, 2012). Sin embargo, es una estrategia que tiene el videojuego para concretar y orientar la acción introduciendo al usuario en el contexto material del juego -lo implica a través de un personaje- y se evita el posible caos visual y fatiga que puede generar la falta de referencia en el espacio lúdico. En los casos seleccionados se cumple la característica de que, en principio, quien lidera es uno más de la multitud. Es decir, en ningún caso deja de ser crowd simulation y el control se mantiene integrado en el medio.

Okhlos (Coffee Powered Machine, 2016) es un título ambientado en la Grecia clásica, donde los dioses del panteón helénico existen y gobiernan a la humanidad bajo un sistema represivo. La humanidad se harta de esto y se une para revertir la opresión acabando con el gobierno de los dioses. El objetivo es, a lo largo de diferentes escenarios, reunir a más individuos para sumarlos al colectivo y enfrentarse a diferentes dioses a modo de "jefes finales" o subalternos para acabar con su gobierno sobre la humanidad.

La multitud se compone de "filósofos", "soldados", "civiles" y "esclavos", en una forma de intentar representar las divisiones sociales de la época -sin ninguna ambición de ser históricamente exacto-. Cada elemento tiene habilidades específicas: los esclavos recogen objetos para usarlos contra los enemigos; los soldados infligen más daño o defienden al grupo; los civiles, aparte de luchar como los demás, son un elemento de cambio para canjear en algunos momentos por elementos de los otros grupos. El papel especial lo tienen los filósofos, que son los encargados de "liderar" la multitud. El usuario "controla" a un filósofo, a través del cual guía al grupo; establece dónde se mueve la multitud, cuándo atacar y cuándo defenderse. Es un componente más; si muere, es sustituido por otro filósofo del grupo. Su papel 
es importante en la medida en que, si el grupo se queda sin filósofos, la partida termina, pues no hay quien guíe.

Tonight we riot (Pixel Pusher Union 512, 2020) tiene una estructura muy similar a la de Okhlos pero profundiza más en el anonimato de quien guía a la multitud. En esta obra, ambientada en un mundo industrial, una mega-corporación controla y oprime a la población a través del trabajo, en una especie de síntesis estatal-policial-empresarial que domina todos los aspectos de la vida. Las diferentes pantallas comienzan con un pequeño grupo de trabajadores, que tienen que ir avanzando por el escenario urbano e industrial "tomando las calles": liberando a trabajadores de las fábricas que se unen al grupo, enfrentándose a la policía y diferentes grupos represivos, y, por último, tomando un banco o enfrentándose a un jefe que marca el final de la pantalla. El objetivo es vencer al malvado Estado-empresa y constituir una sociedad emancipada.

El usuario controla a la multitud a través de uno de sus componentes. Éste no tiene ninguna característica visual diferente del resto del grupo, aunque sí se les individualiza en el retrato diferente que cada uno tiene cuando pasan a liderar. Tampoco cuenta con ninguna habilidad especial que haga de él un personaje más importante que el resto. Es uno más del grupo que lidera la acción y ataca con ella, y en caso caer en la refriega contra la policía, es sustituido por cualquier otro componente del grupo. Su papel se centra en hacer avanzar o retroceder a la multitud y, dependiendo del contexto, usar contra la policía y su armamento diferentes elementos: adoquines, máscaras de gas, cócteles molotov, etc. En este caso, la multitud desaparece -el juego termina- cuando muere el último de los componentes del grupo.

Tooth an tail (Pokewatch Games, 2017), a diferencia de los títulos anteriores, es un caso especial debido a la propia complejidad del trasfondo del título. Este videojuego se sitúa en un mundo de animales antropomorfizados donde cuatro facciones muy estereotipadas compiten por la supervivencia en una sociedad que se encuentra en quiebra. Cada una de estas facciones representa a "estamentos" de la sociedad: los "abrigos largos" representan a una mezcla entre burguesía y aristocracia; la "gente común" serían la masa trabajadora pobre; la "KSR", facción militar, formada principalmente por el ejército; y los "civilizados", una especie de facción teocrática asociada al poder central. Cada facción tiene sus propias reivindicaciones: burgueses, militares y religiosos, buscan, cada uno a su manera, imponer un sistema autoritario que beneficie sus intereses y minimizar el poder del resto; la gente común, por su parte, busca librarse de la opresión del resto de facciones y constituir una sociedad emancipada. Lo que mueve a todos en esta "guerra civil" es la escasez de comida.

La acción se desarrolla en diferentes escenarios donde el usuario tiene que conseguir objetivos "abanderando" un grupo de "rebeldes". El usuario sólo controla al estandarte, cuya única tarea es ordenar y gestionar los recursos con los que se "alimenta" la multitud. Ésta es un grupo "especializado", con diferentes unidades con habilidades especiales representadas por diferentes animales. Éstos serían el cuerpo social, los miembros individuales de las diferentes facciones que luchan en común contra las otras facciones con los objetivos concretos de cada una. De este modo, el abanderado no aparece como una "personalidad" que haga su voluntad sino como el líder accidental de un proyecto común. La victoria o la derrota en cada escenario vienen definidas por el ejercicio del dominio de una facción sobre otra en términos puramente estratégicos.

\subsection{Multitud agente}

El diseño de dinámicas de multitudes es complejo, por eso en los casos citados hasta ahora se ha preferido enfocar el "control" de la multitud desde una singularidad inserta en ella. Si hay que atenerse a la definición de multitud, esto no describiría la acción real concreta de la 
multitud. Son pocos los casos donde la multitud actúa como un todo manteniendo las particularidades posibles de acción dentro de ella y que sea controlada desde dentro, es decir, sin individualización y con la acción de todo el colectivo de forma dinámica. En los ejemplos que siguen este patrón tiene una mayor presencia la actualidad política.

Anarcute (Anarteam, 2016) presenta un mundo controlado por un macro-Estado policial represivo, y la misión del usuario es destruir este sistema, como en otros casos. La diferencia es que, aparentemente, los escenarios donde se realizan las misiones están situados en grandes ciudades actuales, como París o Tokio. El desarrollo es similar al de Okhlos o Tonight we riot: recorrer un escenario reuniendo nuevos elementos para la multitud y usarlos contra objetivos concretos. En este caso, se parte con un pequeño grupo y se encuentran grupos "dormidos" a lo largo del escenario, mientras se sortean o se enfrentan grupos policiales de diferentes clases, trampas, o pequeños puzzles que requieren una determinada cantidad de gente en la multitud.

Los escenarios se superan cumpliendo una serie de condiciones como terminar con un cierto número de individuos en la multitud, tomar o destruir ciertas posiciones o derrotar a un jefe final. Esto depende siempre de la cantidad de individuos que se controle en la multitud, que se guía como un grupo unitario, aunque cuida sus dinámicas: los individuos se mueven a diferentes velocidades, alguno se puede quedar rezagado, o no responder exactamente a las instrucciones del usuario. No hay un foco que organice el grupo más que por un vago centro en torno al cual se amontonan. No tienen habilidades especiales -en cualquier caso, todos son capaces hacer lo mismo-, ni destacan los diferentes miembros más que por la apariencia (animales antropomorfizados); funcionan como un todo fluido frente a la policía, que, a pesar de la despersonalización habitual, sí actúan individualizados.

Riot: Civil Unrest (Leonard Menchiari/IV Productions, 2019) es, de todas las obras señaladas hasta ahora, el más actual, en términos de una búsqueda de representación de la realidad presente lo más fiel posible. Al igual que Anarcute se sitúa en la actualidad, pero sin ficcionarla: el título se desarrolla en torno a las protestas de los últimos años, como las de la Primavera Árabe -en concreto las de Egipto en la plaza Tahrir-, el 15M, las protestas de la plaza Syntagma en Grecia, o las protestas No TAV en Italia. Sin embargo, a diferencia del resto, en este no se trata de recorrer un escenario para cumplir unos objetivos, sino, a través de modelos reales -en tanto que intentan imitar dinámicas de protestas concretas- configurar patrones de protesta y de control de la multitud más cercanos a situaciones que se pueden conocer por las noticias o por haber participado en manifestaciones. Busca, en cierto sentido, una representación realista.

Las campañas, relacionadas con cada una de las protestas citadas, se componen de diferentes escenarios tomados del desarrollo concreto de las protestas. Por ejemplo, con el 15M aparece la Puerta del Sol o la Plaça Catalunya. En estos escenarios, la multitud de manifestantes son, en principio, un reflejo de la composición heterogénea de las protestas reales. También, a diferencia de las obras anteriores, la multitud no se lidera o controla, sino que el usuario, como multitud, realiza acciones colectivas, como puede ser que los miembros de la multitud se agrupen en un punto, que se suba una foto o un mensaje a las redes sociales para llamar a más gente, defenderse de los ataques de la policía, etc. Pero en ningún momento es el usuario quien mueve a la multitud; ésta, incluso, puede salir en desbandada como reacción a la policía al mismo tiempo que se tata de agruparla.

Una novedad con respecto al resto de títulos (tal vez no en relación a Tooth and tail), en ese espíritu de realismo, es que se puede controlar además a la policía en el acto de reprimir las protestas. Aunque sus mecánicas son diferentes también se controla como un todo, en este caso mucho menos individualizado, pero manteniendo la deshumanización habitual de la policía. Los objetivos, definidos en cada escenario como mantener una posición en la plaza frente a la 
policía, o expulsar a los manifestantes, cada grupo usando todos los medios que tienen a su disposición -de medios pacíficos a violentos-, dependiendo de cómo se consigan definen si en escenarios siguientes la policía es más violenta, hay un mayor número de manifestantes, etc. Aunque difusa en el contexto, la finalidad es, o conseguir realizar las reclamaciones de los manifestantes, o reprimirlos.

\section{CONTRADICCIONES DE LA REPRESENTACIÓN DE LA MULTITUD}

Tratar a la multitud como un sujeto propio en términos políticos pretende dotarlo de una agencia que, por lo general, no tiene (porque es difícil dotar de personalidad a una pluralidad). El efecto suele ser el contrario. Por lo general, la multitud aparece cosificada, instrumentalizada por unos objetivos concretos. Excepto en el caso de Tonight we riot (que sería un arcade de scroll lateral), el resto de títulos se ajustan al modelo RTS [real time strategy], lo cual concreta bastante la concepción de la misión y objetivos de la multitud: hay objetivos concretos que hay que alcanzar para considerar la misión un éxito, y para ello la multitud tiene que seguir estrategias concretas que lleven al éxito, como una planificación militar en el campo de batalla. Es decir, para la multitud no hay objetivo político, hay objetivo táctico (inmediato, funcional).

De este modo, la relación con el espacio (la calle, la plaza, los edificios) es táctica: hay que mantener/tomar esta posición para ganar. Del mismo modo, la relación interna de la multitud como multitud se encuentra completamente de-subjetivada: son multitudes heterogéneas y con un mínimo de individualización, pero no son una pluralidad unida. Hay un muchos, pero no un muchos en tanto muchos. Cuando la multitud es tratada como sujeto con voluntad se despersonaliza y lo único que queda es la acción presente en forma de violencia.

\subsection{El espacio y la lucha}

El contexto y el trasfondo de los videojuegos es un elemento importante de inmersión para el usuario. Sin embargo, a pesar del contenido político claro y expreso de todos los títulos, raramente afecta a las mecánicas. Salvo momentos puntuales, no toman una posición explícita ni un compromiso concreto que, a la postre, afecte a las mecánicas de juego y a la forma del usuario de relacionarse con el mundo videolúdico a través de esa multitud que exprese a la multitud.

En el caso de la multitud como ostentadora de unas reivindicaciones políticas, la relación con el espacio es fundamental para entenderlas, porque se presupone que, frente al poder -el Estado, el Imperio, impersonal, como una fuerza deslocalizada-, la multitud es la población concreta de un determinado espacio, y cuyas reivindicaciones se enfocan en la vida en ese espacio. Es el caso de Tonight we riot, o más livianamente en Okhlos o en Anarcute: el espacio ha sido ocupado por fuerzas que coartan la vida, y hay que subvertir ese dominio. Excepto en Tooth and tail, predomina el ámbito urbano, que es el propio de la heterogeneidad que clama la multitud; en Okhlos aparece la ciudad "grecolatina" en su generalidad, pero para el resto de títulos el medio es una ciudad contemporánea donde predomina el asfalto y los edificios altos.

Sin embargo, la relación efectiva con el espacio excluye por completo el contexto del que hace a la multitud partícipe. El espacio en Tooth and tail es un simple campo de batalla. Por su parte, a pesar de la importancia que da Tonight we riot a la lucha como forma de "liberación", la relación con la calle es meramente accidental, es "donde se da la lucha”. Sí es capaz de señalar, aunque sea desde una perspectiva estereotipada, las relaciones de poder en el medio urbano represivo: los trabajadores que se pueden unir a la multitud están en fábricas que son "liberadas", los enemigos están en los bancos, y la policía entre la multitud y ellos. Sólo 
en Riot se da, en la estrechez de su contexto, una relación mínimamente orgánica: la plaza es "nuestra" y "no nos echan".

Por otra parte, el hecho de que en Okhlos o en Anarcute se puedan destruir edificios hasta sus cimientos dice poco de la multitud como habitantes del lugar. En el primer título simplemente son arrasados al paso del grupo; en el segundo, la destrucción de edificios está condicionada a la estrategia y la cantidad de miembros que compongan la multitud: si hay suficientes y para llegar a un lugar sorteando a la policía hay que tirar un edificio, se hace sin ningún tipo de penalización. Lo que sugiere es que, o no hay relación de la multitud con su entorno -lo cual es dudoso porque los miembros se encuentran dispersos por el mapa-, o es irrelevante esta relación. Las reivindicaciones políticas quedan en segundo plano, lo cual afecta más al caso de Anarcute que a Okhlos.

También ocurre con las "herramientas" para la revuelta que la multitud encuentra a su paso. En Tonight we riot los materiales que se usan como armas se encuentra en la calle, a disposición de los revoltosos, y sería mucho suponer que otra persona las ha puesto ahí para ellos -se puede suponer con los adoquines, no con los cócteles molotov-. Tanto en Anarcute como en Okhlos, la multitud encuentra cosas mientras arrasan el escenario. Los miembros de la multitud de Anarcute cargan con señales, cubos de basura, trozos de marquesinas, que después se usan contra la policía. En Okhlos ocurre lo mismo con recursos acordes al contexto. Es, hasta cierto punto, razonable: si se cruza una aldea, se encontrará comida; y si se arrasa unos barracones, se encontrarán armas. Pero funciona como un saqueo que desplaza completamente el centro de las reivindicaciones de la multitud como sujeto político a la violencia sin fondo.

Llegados a este punto, hay que hacer una aclaración: Okhlos en todo momento habla de mobs (turbas), no de crowds. Esto resulta relevante en relación al propio título de la obra: okhlos es una palabra griega que se suele traducir como "muchedumbre" o "turba". En la antigüedad era usado como sinónimo de "democracia" como degeneración del sistema virtuoso de los muchos que sería la "república". La "oclocracia" sería el "gobierno de la muchedumbre" o el "poder de la turba" [mob-rules], y teóricamente tendría su origen en una mezcla de hybris o "soberbia" y paranomía o "ilicitud": sería la soberbia de la plebe de creerse capaz de gobernarse a sí misma y, por ello, violar la justicia y la legalidad (Padilla Gálvez, 2017: 170).

La multitud de Okhlos constituiría algo más parecido a la multitud pre-industrial de Rudé: un conglomerado espontáneo y explosivo con un objetivo claro -acabar con la tiranía de los dioses- pero poco orientado organizativa y políticamente. Hay un componente fuerte de hybris en tanto que soberbia trágica de rebelión contra lo dispuesto por el destino -el mandato de los dioses-, pero no se aprecia paranomía desde el momento en que no se trata en ningún momento de un levantamiento contra el destino sino en favor de una justicia negada. Es decir, se trata desde una mentalidad rebelde moderna, donde la diferencia con la "turba" es que mantiene un proyecto constituyente (fundar una sociedad sin la tiranía de los dioses) y permite profundizar en él a través del conflicto. Aunque todo esto afecta de forma directa a Okhlos, es algo que se refleja en las reivindicaciones del contexto o el trasfondo del resto de títulos, que encuentran un desequilibrio entre teoría y práctica.

El caso de Tooth and tail se encuentra en el límite de lo relevante para este trabajo. Es interesante desde el momento en que quiere representar a diferentes grupos sociales con intereses diferentes compuestos por una gran variedad individualizada de miembros pero que actúan como cuerpo común en sus intereses; especialmente en las facciones de los "abrigos largos" y la "gente común", donde no existe en principio una jerarquía y una organización que se supone en las facciones militar y religiosa. Se puede observar una lejana referencia a la Revolución rusa de 1917, donde diferentes poderes se disputaron el poder como una forma de 
hegemonía, con el hambre y la carestía de la vida de fondo y como condición de todo movimiento. Pero entonces lo que queda es guerra civil, lo cual escapa de la dinámica de la multitud.

Los casos de Anarcute, Tonigth we riot y Riot se presentan políticamente más cercanos al presente, y la muchedumbre pierde peso en ese movimiento explosivo y destructor y mantiene otra tensión con sus reivindicaciones, aunque no satisfagan las exigencias políticas más allá de la táctica. Esto se debe a que centran su temática en el conflicto con un Estado represivo que tiene su trasunto en acontecimientos históricos (en tanto que realmente ocurridos); más liviano y simbólico en Anarcute y Tonigth we riot, más explícito en Riot. El comportamiento con el enemigo policial-represivo y con el entorno se homogeiniza: todo pasa a ser objeto de represión desde el momento que la represión ocupa todo el espacio, por eso se vuelve lícito derribar edificios, por ejemplo, para abrir camino. En cualquier caso, el problema de la autoridad policial y su carácter represivo dentro de los mecanismos de control del Estado no pertenece al ámbito de la multitud, sino al Imperio. La brutalidad policial es un elemento más de su constitución, y es sobre algo que se ha escrito en profundidad (Carter, 1975: 69 y ss.; della Porta y Reiter, 1998; Oliver y Urda, 2015: 57 y ss.).

Es otro tipo de autoridad la que pertenece a la multitud, y es la que queda en entredicho en las obras señaladas. La "lucha" queda en todo momento suspendida por la táctica. Por ejemplo, la tensión política en Tonight we riot es meramente conflictiva directa. Aquí la intención no busca la sutilidad de la pluralidad sino el combate de la multitud. La heterogeneidad queda supeditada al objetivo global; la multitud se mueve como un cuerpo común, con un objetivo común, y no como una "pluralidad común". Algo similar ocurre en Anarcute, con una mayor individualización, pero con la misma reducción al estereotipo de la lucha contra el Estado represivo. A esto se le añade que el apartado "cute" -que completa el título- frivoliza con el tema.

Mucho más centrado está Riot, también porque su contexto estrecha mucho las posibilidades de desatino. Aquí el trasfondo concreto acota las necesidades de la marcha de la multitud. No se enfrenta a una campaña en la cual tiene que ir progresando, sino al mantenimiento de una protesta con un desarrollo político concreto que, en mayor o menor medida, no requiere de la multitud, aunque sí se ve afectado por la multitud. Por eso, y por tener a los ejemplos reales como apoyo, la estructura que toma la multitud en las diferentes plazas o enclaves que ocupan se asemeja más a la dinámica propia de una protesta ciudadana genuina. El control, como ya se ha señalado, también da pistas de la irregularidad de su comportamiento, de las inercias. Sin embargo, esto cambia y se vuelve una extorsión de las dinámicas propias al situarse en el interior de la multitud como agente, y no como elemento con respecto a un espacio o una lucha.

\subsection{El cuerpo político}

Por lo general, los estudios que se han referido al "cuerpo" y al tratamiento del mismo en el ámbito videolúdico se han centrado en temas de género y sexualidad -la literatura al respecto es extensa (Jenkins, 1998; Healey, 2016; Barr, 2018: 91 y ss.)-. Se ha estudiado la forma de representar el cuerpo en su individualidad diferencial, en su diversidad (de los NPC's genéricos que rellenan el mundo a los "creadores de personajes"). Sin embargo, hay pocas referencias dentro del videojuego a la representación del cuerpo en la sociedad, como parte del cuerpo social. Se parte de la idea de que el cuerpo social condiciona la forma en que se percibe el cuerpo físico (Douglas, 1988: 89), pero en el videojuego no suele mostrar la forma en que el cuerpo físico pertenece al cuerpo social. Es decir, se reconocen los mecanismos mediante los cuales el género o la "raza" son representados, y cómo individualmente se integran en la 
sociedad, pero la representación se suele volver difusa cuando se trata de integrar los individuos en lo "colectivo".

La inserción del individuo en la sociedad no implica lo mismo en el plano representativo que la concepción global de los individuos como cuerpo político. El cuerpo político -como conjunto de individuos sujetos a un mismo dominio- se encuentra mediado y condicionado por el poder en su "regulation, surveillance, and control of bodies (individual and collective) in reproduction and sexuality, in work and in leisure, in sickness and other forms of deviance and human difference" (Scheper-Hughes y Lock, 1987: 7-8). El poder actúa sobre la heterogeneidad como si la multitud no fuera "multitud"; en eso se basa la regulación, vigilancia y control, y es un problema que ha recorrido toda la Modernidad (Beccaria, 2008: 78; Burke, 2003: 94). De esta forma, la óptica desde la cual se observa la multitud condiciona la forma de representar(se) en ella. En este aspecto, todas las obras señaladas manifiestan las mismas carencias.

La cuestión es la siguiente: la multitud se observa en todo momento desde la (o)posición con respecto al poder, no como contrapoder, que es lo que Negri y Hardt sugieren que constituye a la multitud. La "oposición" no es contrapoder, es "disidencia", y aunque en todo momento aparezca desde la fórmula de rebeldía moderna legitimada por la resistencia a la tiranía de los gobernantes -tema central en el pensamiento político moderno (Negro Pavón, 1992)-, la multitud no aparece como "constituyente", sino como una rebelión que mantiene una contradicción como "ilegitimidad legítima": la multitud tiene razón, pero el poder está legitimado por su naturaleza a usar la fuerza contra los revoltosos. De ahí la deriva a la táctica y la pobre representación de la heterogeneidad del cuerpo político de la multitud.

Por ejemplo, el detalle más relevante de Tonight we riot es que no se hace distinción alguna entre quien compone la multitud. Se ha elegido una estética muy definida entre el grupo general de los trabajadores rebeldes (ropa gris, gorras de tela, pañuelos rojos) como la imagen tradicional y estereotipada del revolucionario urbano e industrial. Para la multitud de trabajadores rebeldes se juega con una contradicción interesante: la obra recuerda desde el principio que son individuos con sus pasiones y deseos individuales, y que es el sistema el que los ha convertido en algo homogéneo. Se rebelan para volver a ser individuos. Por su parte, en Tooth and tail y Okhlos hay una pluralidad expresada con acierto desde el primer momento, pero, una vez más, queda como necesidad táctica y no política. Algo similar ocurre en Anarcute, donde la heterogeneidad es meramente estética.

Riot es, posiblemente, la obra donde se concentra con mayor precisión la contradicción que atraviesa toda la representación de la multitud como multitud agente en las obras señaladas. En Riot hay multitud, pero no una multitud humana. Para el usuario se actúa como multitud inmerso en la acción a través de una violencia despersonalizada, despolitizada, a pesar del contexto en el que quiere enmarcarse, porque, aunque los textos digan otra cosa, en el "combate" (concreto, el que se juega) no hay compromiso, sólo combate. Sus propuestas son de base polémicas, pero no polemiza; es un letrero de presentación para ubicar la acción, pero no para desarrollar la acción. Ahí radica todo el problema de la representación y la acción de la multitud en los videojuegos: no ser político con un tema básicamente político.

La inclusión del término "cuerpo político" no es arbitraria. Se habla de la multitud como cuerpo sujeto a derechos, como "persona colectiva", porque, al contrario, el "cuerpo" (lo físico) tiene valor jurídico, pero no es sujeto de derecho. Lo que tiene el ciudadano, a la postre, es su cuerpo (grado cero del derecho); se tiene un cuerpo que mostrar (habeas corpus), pero un cuerpo no es una "persona" (García López, 2018a: 666), y es lo que queda en la representación de la multitud: hay cuerpos, no personas. El cuerpo es lo que se sacrifica en las protestas. Sin embargo, la reivindicación moderna del cuerpo como dispositivo contenedor de la personalidad está tan vacía en la doctrina legal como en estos títulos. El derecho protege a la 
persona y a las cosas de la persona, no a las cosas en general.

La manifestación de esta dicotomía es clave en los videojuegos, Por ejemplo, cuando se comete un "asesinato" en diferentes obras, el cadáver resultante es motivo de alarma, pero no de preocupación "personal": lo que queda es un cuerpo, no una persona; quien encuentra el cadáver se preocupa por su seguridad, no por la persona que ha fallecido; nunca se llama a un médico por si se puede salvar la vida. Del mismo modo, los compañeros caídos en la multitud son pisados por el grupo sin demostrar conciencia alguna de su debilidad o vulnerabilidad: se desprenden miembros, no personas. Es sorprendente por su contenido político este olvido cuando en otras obras sí se recuerda a los caídos, a través de instancias como el "cementerio".

Todas las obras olvidan que la persona es sujeto de derecho en favor de las dinámicas de multitudes, es decir, hay una reducción de la multitud como sujeto político a la dinámica de los cuerpos como colectividad física. La persona incluso en la transgresión de la ley está protegida de la arbitrariedad por la propia ley, y no pierde esos derechos al entrar a formar parte de la multitud, pero esto no incluye al cuerpo, por eso se permite esa contradicción y ese "vacío ético" que licita la violencia. Al mismo tiempo, "la vida desnuda se convierte en la categoría ética que cuestiona los sistemas jurídico-políticos y económicos, así como sus instituciones" (García López, 2018a: 670): la inanidad de la persona como cuerpo en el videojuego no hace más que ser un fiel ejercicio de ideología dominante.

La multitud en las obras señaladas deviene "cosa", no persona, por eso se puede excluir del derecho e incluso de la moral creando un espacio de violencia sin sustento metafísico. A pesar de la contextualización -que es importante en todos los títulos-, la violencia tal y como se representa busca ser neutral, porque la multitud política es muchedumbre, y la "multitud" policial es derecho. En este contexto se podría abrir el espacio de reflexión, como dice Roberto Espósito, entre la "cosa" y la "persona", "lo que ha sido excluido porque es ajeno al binomio entre persona y cosa, es precisamente el elemento que permite el tránsito de una a otra" (2016: 12). Aquí es donde propone abordarlo desde el "cuerpo", y aquí es donde se podría abrir el espacio de reflexión de estos videojuegos que te permiten controlar y ser parte de la multitud, porque es la misma contradicción que se vive cuando el ciudadano como manifestante se sitúa delante de ese otro ciudadano como policía que es representante del poder estatal. Sin embargo, como se ha visto, ese espacio queda cerrado.

\section{CONCLUSIONES}

La representación resulta bastante insatisfactoria, o, al menos, irregular, sea porque la multitud es instrumentalizada (Tooth and tail), o porque el contexto que apoya la representación se trivializa y lo que queda es sólo la violencia. Las mecánicas y dinámicas de la multitud en tanto "colectivo" son satisfactorias, especialmente en Okhlos y en Riot (y en menor medida en Anarcute). Sin embargo, la representación de la multitud en tanto multitud se encuentra extorsionada en todo momento. No es necesario entrar en la estrategia política concreta de la multitud, sólo entender estas diferencias para saber cómo nos representamos en ella. La descripción es efectiva y se traduce de forma satisfactoria al videojuego, pero sólo si se habla desde fuera de la multitud, es decir, desde el usuario que la controla y la ve como un ente ahí sin conexión con su conciencia. No tiene, en su interior, la vida que se le otorga según su definición. Eso retrotrae la idea de multitud al de "muchedumbre" o "turba", lo cual para Okhlos o Tooth and tail puede no ser un problema, pero para el resto sí.

Por lo tanto, atendiendo a los objetivos señalados, en todas las obras, en mayor o menor medida, se da conceptual o mecánicamente la unión de una pluralidad heterogénea con 
diferentes dinámicas (más acusado en Okhlos, mucho menos en Tonight we riot, por ejemplo); $\mathrm{y}$, en mayor o menor medida, se da la articulación de la multitud como "contrapoder", como una forma de resistencia contra un poder dominante -opresivo- (algo presente en todos). Sin embargo, la configuración en torno a unas reivindicaciones de carácter político, social, económico, etc., de tipo estructural, que van más allá de la mera violencia y destrucción del entorno, lo que los diferenciaría de la representación de la "turba", está en entredicho.

En todos los videojuegos señalados (y que es extensible a otras obras que escapan de los límites de este trabajo) hay una incomprensión profunda de su material: "El derecho, mediante la ley, se ocupa de los sujetos, mientras que la política, por medio de la norma, de los cuerpos. Es en este contraste entre el sujeto y el cuerpo, el derecho y la política, la ley y la norma, donde se ubica la violencia" (García López, 2018b: 227). La reducción casi unánime a la violencia en tanto táctica rebelde (o incluso revolucionaria) de esas multitudes que representan confunde los términos sobre los que trabaja. Lo que se supone que es participación polémica en un tema político progresista, es decir, la coordinación de los sujetos como multitud en la revuelta contra un sistema opresivo, desaparece en una gestión reaccionaria de los cuerpos unidos en multitud. La incomprensión de estos elementos en la conceptualización de la multitud jugable -que en ningún momento participa de una discusión entre el derecho y la política- es lo que abre el espacio de la violencia sin solución crítica, y es lo que, al igual que en la política real, depotencia el significado y el papel de la multitud en nuestra representación cotidiana.

\section{BIBLIOGRAFÍA}

Allen-Perkins Avendaño, D. (2012). "Liderazgos informales en el movimiento 15m en Cáceres. Aproximación a su estudio a través del proceso normativo de la asamblea de la ciudad". Revista Estudios, 2, en http://estudios.cnt.es/liderazgos-informales-en-el-movimiento-15m-en-caceresaproximacion-a-su-estudio-a-traves-del-proceso-normativo-de-la-asamblea-de-la-ciudad//Consulta 22/06/2020]

Barr, J. (2018). Video gaming in science fiction. A critical study. Jefferson (NC): McFarland \& Company, Inc., Publisher.

Beccaria, B. (2008). De los delitos y de las penas. Madrid: Alianza.

Bruneau, J., Olivier, A.-H., Pettré, J. (2015). "Going through, going around: A study on individual avoidance of groups". IEEE Transactions on Visualization and Computer Graphics, 21, 4 (Abril): pp. 520-528, DOI: https://doi.org/10.1109/TVCG.2015.2391862

Burke, E. (2003). Reflexiones sobre la Revolución en Francia. Madrid: Alianza.

Carter, A. (1975). Teoría política del anarquismo. Caracas: Monte de Ávila Editores.

Christoph, K.; Dorothée, H.; Peter, V. (2009) "The video game experience as true identification: A theory of enjoyable alterations of players' self-perception”. Communication theory, 19, 4: pp. 351-373, DOI: https://doi.org/10.1111/j.1468-2885.2009.01347.x

Dean, J. y Passavant, P. (2003). The Empire's new clothes: Reading Hardt and Negri. Londres: Routledge, DOI: https://doi.org/10.4324/9780203644003

Della Porta, D. y Reiter, H. (1998). Policing Protest: The Control of Mass Demonstrations in Western Democracies. Minneapolis: University of Minnesota Press, DOI: https://doi.org/10.5749/j.ctttv1tv

Douglas, M. (1988). Símbolos naturales. Exploraciones en cosmología. Madrid: Alianza.

Esposito, R. (2016). Las personas y las cosas. Buenos Aires: Katz, DOI: https://doi.org/10.2307/j.ctvm7bc5k

García López, D. J. (2018a). "Has de tener un cuerpo que mostrar: grado cero de los Derechos Humanos". ISEGORÍA. Revista de Filosofía Moral y Política, 59 (julio-diciembre): pp. 663-682, DOI: https://doi.org/10.3989/isegoria.2018.059.16

García López, D. J. (2018b). "El fin de todos los derechos: el cuerpo viviente como umbral de la 
democracia”. Anales de la Cátedra Francisco Suárez, 52: pp. 223-247.

Hardt, M., y Negri, T. (2002). "La multitud contra el Imperio". Revista OSAL, Observatorio Social de América Latina, 7 (Junio), Buenos Aires: CLACSO (Consejo Latinoamericano de Ciencias Sociales), pp. 159-166.

Hardt, M., y Negri, T. (2004). Multitud. Barcelona: Debate.

Hauserwas, S. (2003) Dissent from the homeland: Essays after September 11. Durham: Duke University Press, DOI: https://doi.org/10.1215/9780822385059

Healey, G. (2016). "Proving grounds: performing masculine identities in Call of Duty: Black Ops". Game Studies, 16, 2 (Diciembre), en http://gamestudies.org/1602/articles/healey [Consulta: 22/06/2020]

Hefner, D., Klimmt, C., Vorderer, P. (2007). "Identification with the player character as determinant of video game enjoyment”. En Ma L., Rauterberg M., Nakatsu R. (Eds.) Entertainment Computing ICEC 2007. ICEC 2007. Lecture Notes in Computer Science, 4740. Berlin/Hedelberg: Springer, pp. 39-48, DOI: https://doi.org/10.1007/978-3-540-74873-1 6

Jenkins, H. (1998). "Complete freedom of movement: videogames as gendered play space". En Cassel, J. y Jenkins, H. (Eds) From Barbie to Mortal Kombat: Gender and Computer Games. Cambridge (Massachusetts): MIT Press, pp. 330-363.

Maïm, J., Yersin, B., Thalmann, D. (2009). "Unique character instances for crowds". IEEE Computer Graphics and Applications, 29, 6 (Noviembre): pp. 82-90, DOI: https://doi.org/10.1109/MCG.2009.129

McDonnell, R., Larkin, M., Dobbyn, S., Collins, S., O’Sullivan, C. (2008). "Clone attack! Perception of crowd variety". ACM Transactions on Graphics, 27, 3: pp. 1-8, DOI: https://doi.org/10.1145/1360612.1360625

Negro Pavón, D. (1992). "Derecho de resistencia y tiranía". Logos. Anales del seminario de metafísica, número extra, Madrid: Ed. Complutense, p. 683-707.

Oliver, P. y Urda, J.-C. (2015). Protesta democrática y democracia antiprotesta. Los movimientos sociales ante la represión policial y las leyes mordaza. Arre, Pamiela.

Ondřej, J., Pettré. J., Olivier, A.-H., Donikian, S. (2010). "A synthetic-vision based steering approach for crowd simulation". ACM Transactions on Graphics, 29, 4 (July), pp. 123:1-123:9, DOI: https://doi.org/10.1145/1778765.1778860

Padilla Gálvez, J. (2017). "Democracy in Times of Ochlocracy”. Synthesis philosophica, 63, 1: pp. 167 178, DOI: https://doi.org/10.21464/sp32112

Rudé, G. (2009). La multitud en la historia. Los disturbios populares en Francia e Inglaterra, 1730-1848. Madrid: Siglo XXI.

Scheper-Hughes, N. y Lock, M. (1987). "The mindful body: A prolegomenon to future work in medical anthropology". Medical Anthropology Quarterly, 1, 1: pp. 6-41, DOI: https://doi.org/10.1525/maq.1987.1.1.02a00020

Thoburn, N. (2003). Deleuze, Marx and politics. Londres: Routledge, DOI: https://doi.org/10.4324/9780203361740

Virno, P. (2003). Gramática de la multitud. Para un análisis de las formas de vida contemporáneas. Madrid: Traficantes de sueños.

Wellmer, A. (1993). Sobre la dialéctica de modernidad y postmodernidad. La crítica de la razón después de Adorno. Madrid: Visor.

\section{LUDOGRAFÍA}

1979 Revolution: Black Friday (iNK Stories, 2016) Web: https://1979revolutiongame.com

Anarcute (Anarteam, 2016) Web: https://anarcute.com

Assassin's Creed (Ubisoft) [serie] Web: https://www.ubisoft.com/es-es/game/assassins-creed

Bloodborne (From Software, 2015) Web: https://www.fromsoftware.jp/ww/detail.html?csm=094

Hitman (IO Interactive) [serie] Web: https://www.ioi.dk/hitman/

Inside (Palydead, 2016) Web: https://playdead.com/games/inside/

Kids (Playables, 2019) Web: https://playkids.ch 
Okhlos (Coffee Powered Machine, 2016) Web: http://coffeepoweredmachine.com/okhlos/

Riot: Civil Unrest (Leonard Menchiari/IV Productions, 2019) Web: http://www.riotgame.org

Tonight we riot (Pixel Pusher Union 512, 2020) Web: https://tonightweriot.com

Tooth an tail (Pokewatch Games, 2017) Web: http://www.toothandtailgame.com

Total War (Creative Assembly) [serie] Web: https://www.creative-assembly.com

Totally Accurate Battle Simulator (Landfall Games, 2019) Web: https://landfall.se/totally-accuratebattle-simulator-early-access

Ultimate Epic Battle Simulator (Brilliant Game Studio, 2017) Web:

https://www.brilliantgamestudios.com

\section{Breve currículo:}

\section{Antonio Flores Ledesma}

Doctor en Filosofia por la Universidad de Granada con la tesis La praxis emancipatoria de la obra de arte en el pensamiento de Theodor W. Adorno. Una investigación de las relaciones entre arte y política en la obra del pensador alemán. Grado en Filosofía por la Universidad de Sevilla. Máster en Filosofía Contemporánea. Su línea de investigación es la estética marxista y la crítica ideológica de la cultura occidental. Ha publicado artículos sobre el realismo soviético y su significación política o sobre la ideología de los videojuegos históricos. Colaborador con medios web como Canino, Nivel Oculto, o AnaitGames. 